Fabbroni, M., A. F. Casas Marty \& H. A. Keller. 2021. Calotropis procera (Apocynaceae), primeras evidencias de su naturalización en Argentina. Bonplandia 30(1): 61-66. http://dx.doi.org/10.30972/bon.3014590

Recibido 27 Septiembre 2020. Aceptado 6 Noviembre 2020.

Publicado en línea: 23 de diciembre 2020. Publicado impreso: 15 de febrero 2021.

ISSN 0524-0476 impreso. ISSN 1853-8460 en línea.

\title{
Calotropis procera (ApocynaCeAe), PRIMERAS EVIdenCIAS de SU NATURALizaCión EN
} Argentina

\author{
Calotropis procera (Apocynaceae), first evidences of its naturalización in Argentina
}

\author{
Mariela Fabbroni ${ }^{1}$, Ariel F. Casas Marty ${ }^{1} \mathbb{D}$ \& Héctor A. Keller ${ }^{2} \mathbb{D}$
}

\begin{abstract}
Resumen: Sobre la base de observaciones efectuadas y muestras recolectadas en el noroeste argentino, se documenta por primera vez para el país, la presencia de una especie adventicia, Calotropis procera (Apocynaceae: Asclepiadoideae) originaria de África y SO Asiático, naturalizada en diversas partes del mundo y con gran potencial invasivo. La especie es descripta, ilustrada mediante fotografías y se brinda información sobre las poblaciones halladas.
\end{abstract}

Palabras clave: Arbustos, Asclepiadoideae, plantas exóticas, Provincia de Salta.

Summary: According to observations and samples collected in northwestern Argentina, the presence of an adventitious species, Calotropis procera (Apocynaceae: Asclepiadoideae), native to Africa and SW Asia, naturalized in various parts of the world and with great invasive potential, is documented for the first time in this country. The species is described, illustrated by photographs, and information on the populations found is provided.

Key words: Alien plants, Asclepiadoideae, Salta Province, shrubs.

\section{Introducción}

La familia Apocynaceae comprende 378 géneros y alrededor de 5350 especies de distribución predominantemente pantropical, con unos pocos géneros alcanzando regiones templadas (Endress et al., 2018). Diversas especies con atributos vegetativos o reproductivos vistosos son cultivadas como ornamentales en todo el mundo, lo cual en muchos casos origina procesos de naturalización. En la Argentina se han documentado seis especies adventicias de esta familia, Asclepias curassavica L. (Hechem et Ezcurra, 2006), A. fruticosa L. (Zuloaga et al., 2019), Catharanthus roseus (L.) G. Don (Xifreda, 1984); Gomphocarpus physocarpus E. Mey. (Zanotti et al., 2020), Plumeria rubra L. (Ezcurra, 2005) y Vinca major L. (Ezcurra, 1981).
El género afroasiático Calotropis R. Br. cuenta con tres especies que se han naturalizado en regiones tropicales de todo el mundo. El progresivo avance de estas especies obedece en parte a la gran capacidad de dispersión de sus diásporas anemocóricas, como así también a su relación con las comunidades humanas que utilizan estas especies y las van difundiendo mediante el intercambio de cultígenos y otros tipos de interacciones sociales (Csurhes et Edwards, 1998; Moro et al., 2012).

Calotropis procera (Aiton) A. W. Aiton es nativa de África, Madagascar, Península Arábica, y del SO asiático (Rahman et Wilcock, 1991) y está naturalizada en diversas regiones áridas del mundo. Habita en sitios abiertos, sabanas, bordes de rutas, caminos $\mathrm{y}$ otros ambientes antropizados, en zonas de baja y media altitud (desde el nivel del

\footnotetext{
${ }^{1}$ Facultad de Ciencias Naturales, Sede Regional Orán, Universidad Nacional de Salta, Salta, Argentina.

${ }^{2}$ Instituto de Botánica del Nordeste - CONICET, Casilla de Correo 209, 3400-Corrientes, Argentina.

E-mails: marielafabbroni@gmail.com; kellerhector@hotmail.com
} 
mar hasta $500 \mathrm{~m}$ ) y sobre suelos secos y arenosos e incluso en orillas de cursos de agua (Sharma, 1934; Csurhes et Edwards, 1998). En América se introdujo en México, gran parte de Mesoamérica (Molina Rosito, 1975; D'Arcy, 1987; Fuentes Fiallo, 2002; Berendsohn et al., 2009; Davidse et al., 2009) y en varios países sudamericanos (Idárraga Piedrahita et al., 2011; Sousa Sobrinho et al., 2013; De Egea et al., 2016). En Paraguay adquiere el porte de árbol pequeño y constituye una maleza de cultivos de difícil erradicación en el Chaco seco (De Egea et al., 2016). En Brasil se volvió invasiva y se extendió por varios estados de la Caatinga, Cerrado y Restinga, debido posiblemente al patrón continuo de floración y fructificación que posee a lo largo del año (Sousa Sobrinho et al., 2013) y en la actualidad se la considera una amenaza para la conservación de la biodiversidad brasileña (Fabricante et al., 2013).

Recientes observaciones de campo $\mathrm{y}$ recolección de material de herbario han permitido dar cuenta de la naturalización en la Argentina de Calotropis procera, lo cual se documenta en la presente contribución. La especie es descripta, ilustrada mediante fotografías y además, se brinda información sobre las poblaciones halladas.

\section{Resultados}

Calotropis procera (Aiton) W. T. Aiton, Hort. Kew. 2: 78. 1811. Asclepias procera Aiton, Hort. Kew. 1: 305. 1789. Madorius procerus (Aiton) Kuntze, Revis. Gen. P1. 2: 421.1891. Calotropis gigantea var. procera (Aiton) P. T. Li, J. S. China Agric. Univ. 12(3): 39. 1991. Typus. Observ. Bot. Icon. 3: t 69. 1768 como Asclepias gigantea (lectotypus designado por Ali, Notes Roy. Bot. Gard. Edinburgh 38: 287. 1980).

Arbustos erectos de hasta 2(-3) $\mathrm{m}$ de altura, ramificados desde la base, de ramas glabras. Hojas sésiles o subsésiles, glaucas; lámina de 14,5-24 × 8-14 cm, elíptica, glabra, con 2-3 hileras de coléteres en la base adaxial de la nervadura principal. Inflorescencias subaxilares, alternas, umbeliformes, con 14-
20 flores; pedúnculo de 4-5 cm de largo, glabro; pedicelos de $2-3 \mathrm{~cm}$ de largo, glabros. Flores con sépalos de 4-5 × 2,5-3 $\mathrm{mm}$, ovados, glabros, cara interna con 2-4 coléteres en el seno. Corola blanca a verdeclara, adaxialmente vinácea en el ápice de los lóbulos, rotácea o subcampanulada; tubo de 5-6 mm de largo, glabro; lóbulos de 5-9 $\times$ 5-6,5 mm, erectos o levemente reflexos en el ápice, ovado-triangulares, glabros. Corona simple, segmentos de 4-5 × 2,5-4 mm, cimbiformes, redondeados y papilosos en el ápice, calcarados en la base, unidos basalmente entre sí por pliegues membranáceos dentados. Ginostegio sésil; anteras rectangulares, situadas transversalmente en el borde superior del ginostegio, alas más largas que el dorso, apéndice apical semilunar; retináculo de 0,42 $0,45 \times 0,12-0,15 \mathrm{~mm}$, oblongo, con expansiones membranáceas laterales; caudículas de 0,30$0,33 \mathrm{~mm}$ de largo, subhorizontales; polinias de 1,20-1,60 × 0,48-0,69 mm, subhorizontales, claviformes. Ápice del ginostegio mamilado. Folículos globoso-recurvados, inflados, de $5-7,1 \times 4-5 \mathrm{~cm}$. Semillas no verrucosas, comosas.

Fenología: En el área de estudio posee un amplio rango de floración-fructificación durante los meses de invierno hasta fines del verano.

Observaciones ecológicas: Es un arbusto alto, ramificado desde la base que rebrota vigorosamente y se ha comprobado su resistencia a la aplicación de herbicida (glifosato) en áreas colindantes a predios productivos (obs. pers.).

Nombres vernáculos: Se desconocen en el área de estudio. En otros países se la conoce como "algodón de seda" (Brasil, Colombia, Cuba, México, Venezuela) (González et al., 2001; Sánchez et al., 2006); "algodón de la playa", "celos", "flor de seda", "globo", "janaúba", "lechero", "quemador", "saco viejo", "seda pintora" (Brasil) (Costa et al., 2009) y "algodón americano", "algodón de Judea", "algodón de playa", "bollo de vieja", "cazuela" y "estrella del norte" (Cuba) (Fuentes Fiallo, 2002). 
Usos: En la zona de Morillo (Salta, Argentina), los lugareños la emplean como planta de jardín en sus viviendas por la facilidad de cultivo, ya que crece espontáneamente en los espacios peridomésticos y porque es una de las pocas plantas con follaje verde y con flores en invierno. En relación al consumo como forraje de animales domésticos, los lugareños difieren en sus opiniones, algunos reportan el consumo de los frutos carnosos por las vacas por su aspecto similar al fruto de la "doca" [Araujia odorata (Hook. \& Arn.) Fontella \& Goyder]. En otras regiones del mundo posee numerosas aplicaciones, en Nigeria es usado como insecticida (Salako et al., 2015) y en Venezuela como nematicida en cultivos hortícolas (González et al., 2001; Sánchez et al., 2006). En Malawi (África) se cita con fines ornamentales (Rodger, 1948) como también en Brasil (Correa, 1939) donde posteriormente se transformó en invasora de pastizales en ambientes secos y semiáridos del NE, Centro-Oeste y SE (Ferreira, 1973; Ferreira et Gomes, 1976). Estudios fitofarmacológicos refieren sus propiedades antipiréticas, analgésicas, antiinflamatorias, antibacterianas, antihelmínticas, purgantes y relajantes musculares (Mossa et al., 1991; Dewan et al., 2000; Shivkar et Kumar, 2003). También como alimento para caprinos (Melo et al., 2001), ovinos y camellos (Nehra et al., 1987) aunque puede ser tóxico para otros animales domésticos según el estado fresco o seco de las hojas y tallos consumidos y del látex (Sharma, 1934; Mahmoud et al., 1979). Sin embargo, es prometedora para la producción animal como alternativa alimenticia en estado fresco o seco (Costa et al., 2009).

Distribución geográfica y hábitat: En la Argentina ha sido hallada creciendo espontáneamente al NE de la provincia de Salta (Fig. 1) en las localidades de Morillo (Dep. Rivadavia) y El Tabacal (Dep. Orán), entre

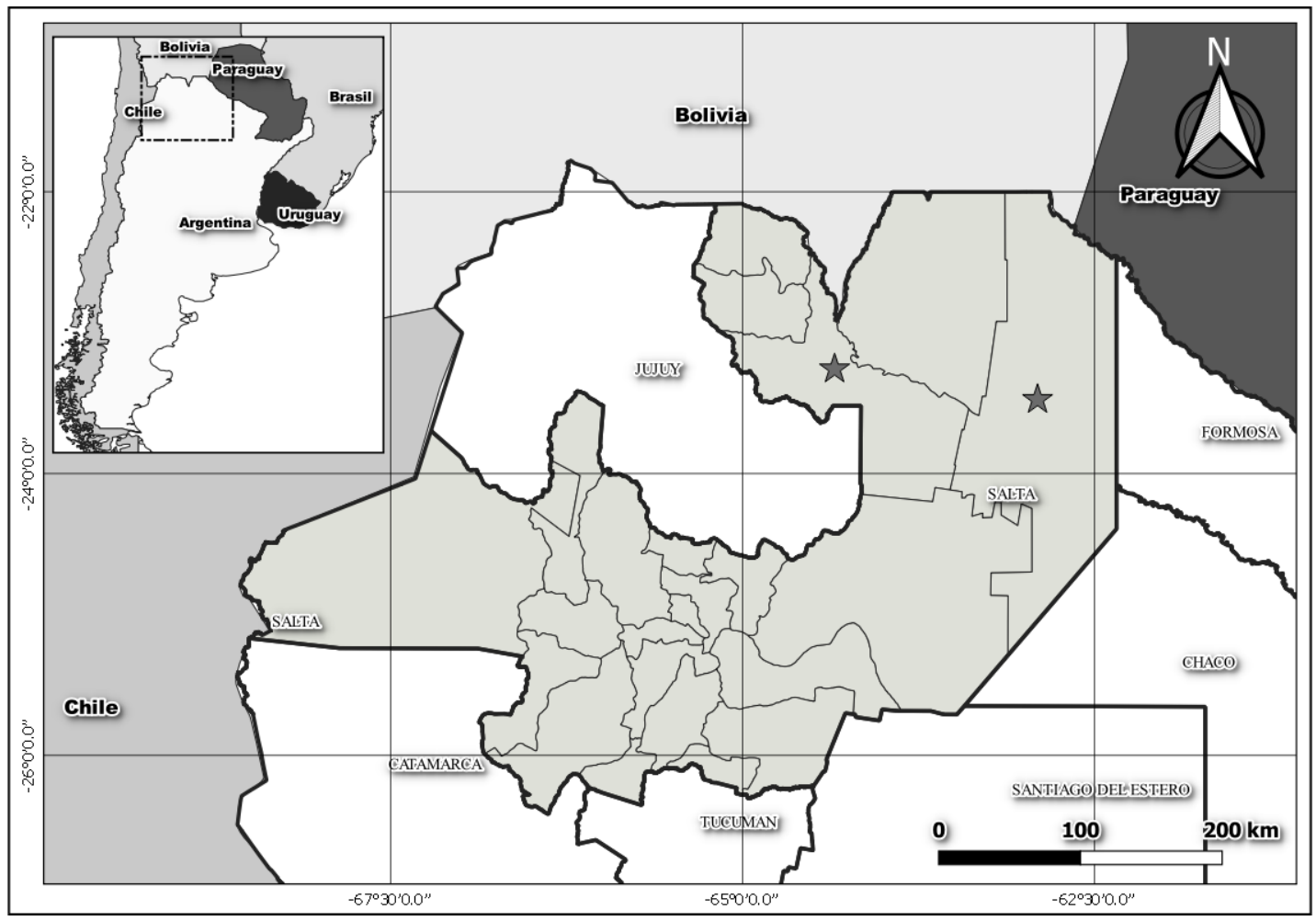

Fig. 1. Mapa de distribución de Calotropis procera en Argentina (estrellas).

Fig. 1. Distribution map of Calotropis procera in Argentina (stars). 
los 200-300 m de altitud. Ambas localidades pertenecen al Distrito Fitogeográfico Chaqueño Occidental (Cabrera, 1976) con un clima semiárido marcadamente estacional y con las precipitaciones concentrándose principalmente en el verano que rondan en promedio entre los 576 y $733 \mathrm{~mm}$ anuales, respectivamente y con una temperatura media anual que asciende a los $21^{\circ} \mathrm{C}$ (Bianchi et Yáñez, 1992).

Calotropis procera se naturalizó en bordes de áreas desmontadas (Fig. 2A), ambientes antropizados como orillas de caminos (Fig. 2B), en los alrededores de las viviendas (Fig. 2C-D), baldios, peladares y bordes de basurales. Crece
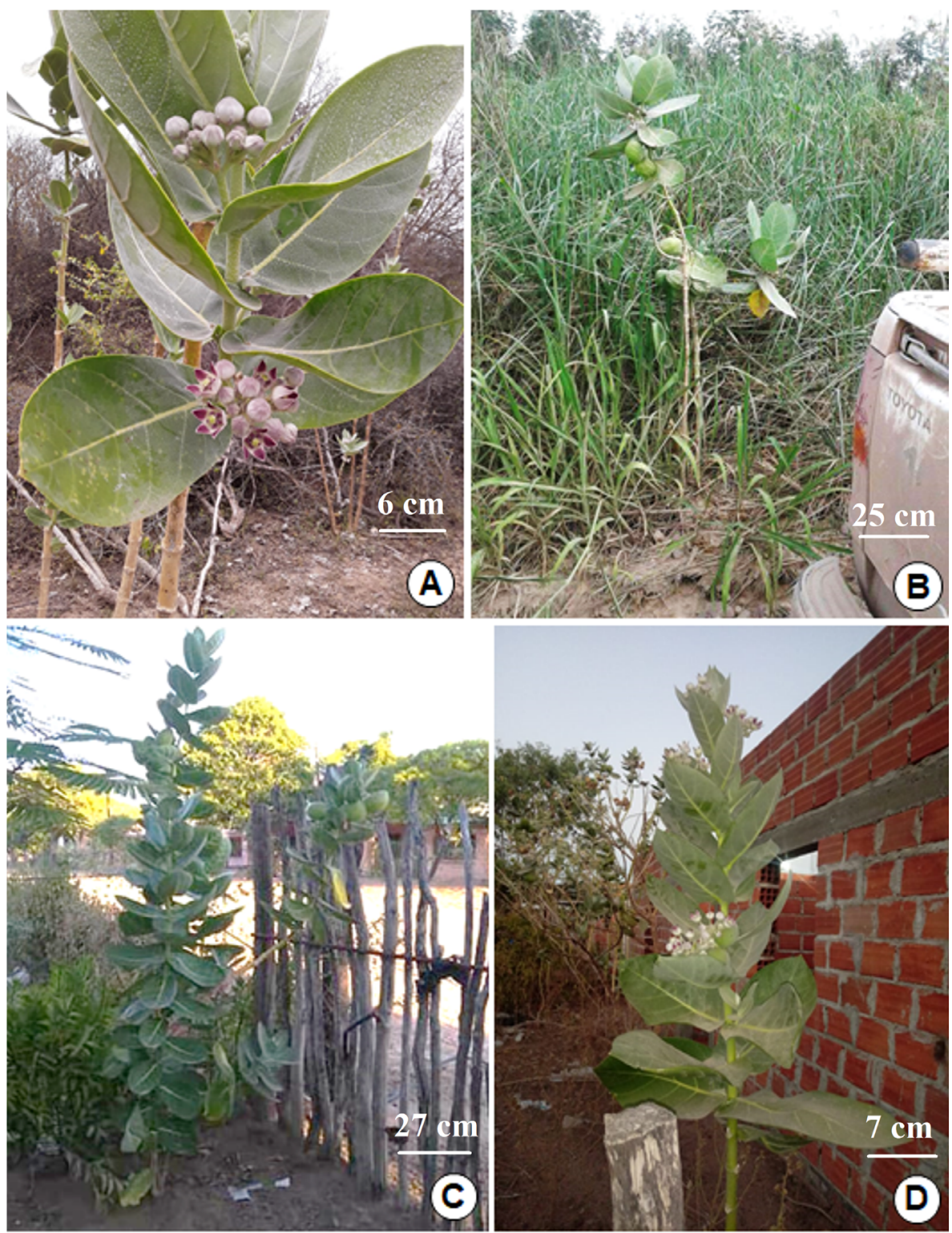

Fig. 2. Sitios donde Calotropis procera crece espontáneamente. A: Borde de bosque chaqueño. B: Borde de caminos. C-D: Alrededor de vivienda.

Fig. 2. Places where Calotropis procera grows spontaneously. A: Next to the Chaco forest. B: Roadsides. C-D: Around houses. 
sobre suelos limoso-arcillosos, algo salinos en peladares, y en suelos arenosos en claros de desmontes recientes en los alrededores del pueblo de Morillo. Ambas localidades son atravesadas por Rutas Nacionales (50 y 81 respectivamente) de alto tránsito vehicular que conectan con la zona fronteriza de Bolivia al $\mathrm{N}$ y con la provincia de Formosa y Paraguay hacia el E. Es posible que la planta haya sido introducida accidentalmente durante los años 2014 a 2016, a través de semillas transportadas en las orugas de las máquinas topadoras y camiones que circulan por la zona, y que se haya extendido en la región por la notable diseminación de sus semillas por el viento.

Material estudiado: ARGENTINA. Salta: Dep. Orán, alrededores de El Tabacal, $300 \mathrm{~m}$, 28-XI-2018, fl, fr, Fabbroni \& Casas 1512 (CTES, MCNS). Dep. Rivadavia, Estación Morillo, Cnel. Juan Solá, 220 m, 10-VIII-2020, fl, fr, Fabbroni \& Paz 1796 (CTES, MCNS). COLOMBIA. La Guajira: Mun. Riohacha, 9-I1944, fl, Haught 3912 (LIL). Magdalena: Mun. Santa Marta, Gaira, 16-IV-1948, fl, fr, Romero Castañeda 1009 (LIL). Santander: orillas del río Chicamocha, 16-XII-1948, fl, Araque Molina \& Barkley 18 S 258 (LIL). MÉXICO. Oaxaca: Mun. Salina Cruz, al lado de la autopista 165, cerca de Salina Cruz, 19-VII-1978, fl, Pennell, Dunn \& Dziekanowski 198 (LIL).

\section{Agradecimientos}

Los autores expresan su gratitud a los revisores anónimos, cuyos comentarios han enriquecido esta contribución. Agradecemos a Alejandra Sofía Paz y Selva Paz, residentes en Morillo, por colaborar en nuestra investigación. Nuestro reconocimiento al personal del Herbario LIL por facilitarnos los ejemplares y al Ing. José L. Rojas (IBONE) por ayudarnos a confeccionar el mapa.

\section{Bibliografía}

Berendsohn, W. G., Gruber, A. K. \& Monterrosa Salomón, J. A. (2009). Nova silva cuscatlanica. Árboles nativos e introducidos de El Salvador. Parte 1: Angiospermae - Familias A a L. Englera 29: $1-438$.
BiAnchi A. R. \& YÁÑez, C. E. (1992). Las Precipitaciones en el Noroeste Argentino. 2da. Edición, INTA EEA, Salta.

Cabrera, A. L. (1976). Regiones Fitogeográficas Argentinas. Enciclopedia Argentina de Agricultura y Jardinería. 2da. Edición. Acme, Buenos Aires.

Correa, P. (1939). Dicionário das plantas úteis do Brasil e das exóticas cultivadas. Imprensa Oficial, Rio de Janeiro.

Costa, R. G., Medeiros, A. N., Rodrigues Alves, A. \& Rodrigues de Medeiros, G. (2009). Perspectivas de utilização da flor-de-seda (Calotropis procera) na produção animal. Caatinga 22: 276-285.

Csurhes, S. \& Edwards, R. (1998). National weeds program, potential environmental weeds in Australia. Candidate species for preventative control. National Parks and Wildlife Biodiversity Group, Environment Australia, Canberra.

D'Arcy, W. G. (1987). Flora of Panamá: Checklist and Index Part. I: The Introduction and Checklist. Monographs in Systematic Botany from the Missouri Botanical Garden 17: 1-328. https://doi.org/10.5962/bhl.title.153180

Davidse, G., Sousa SÁnchez, M., Knapp, S. \& Chiang CABrera, F. (2009). Cucurbitaceae a Polemoniaceae. En Davidse, G., M. Sousa Sánchez, S. Knapp \& F. Chiang Cabrera (eds.), Flora Mesoamericana 4: $1-855$.

De Egea, J., Mereles, F., Peña-Chocarro, M. Del C. \& CÉspedes, G. (2016). Checklist for the crop weeds of Paraguay. PhytoKeys 73: 13-92. https://doi.org./10.3897/phytokeys.73.10135

Dewan, S., Kumar, S. \& Kumar, V. L. (2000). Antipyretic activity of latex of Calotropis procera. Indian Journal of Pharmacology 32: 252.

Endress, M., Meve, U., Middleton, D. J. \& LiedeSchumann, S. (2018). Apocynaceae. En Kadereit, J. W. \& V. Bittrich (eds.), Flowering plants. Eudicots. Apiales and Gentianales (except Rubiaceae). Families and genera of vascular plants 15: 207-411. Springer, Cham, Switzerland. https://doi.org/10.1007/978-3-319-93605-5_3

EzcurRA, C. (1981). Revisión de las Apocináceas de la Argentina. Darwiniana 23: 367-474.

Ezcurra, C. (2005). Apocynaceae. En Anton, A. M. \& F. O. Zuloaga (eds.), Flora Fanerogámica Argentina 91, pp. 54. Proflora y Museo Botánico, Córdoba.

Fabricante, J. R., Nabuco Araújo De Oliveira, M. \& Alves De Siqueira Filho, J. (2013). Aspectos da ecologia de Calotropis procera (Apocynaceae) em uma área de Caatinga alterada pelas obras do Projeto 
de Integração do Rio São Francisco em Mauriti, CE. Rodriguésia 64: 647-654. http://dx.doi.org/10.1590/S2175-78602013000300015

Ferreira, M. B. (1973). Distrito Federal e Goiás sob amenaça de invasora Calotropis procera (Ait). R. Br. Cerrados 21: 20-22.

Ferreira, M. B. \& Gomes, V. (1976). Calotropis procera (Ait) R. Br. uma invasora a ser estudada e controlada no Estado de Minas Gerais. Oreades 5: 68-75.

Fuentes Fiallo, V. R. (2002). Apuntes para la flora económica de Cuba VI. Especies Cauchógenas. Revista del Jardín Botánico Nacional 23: 255-262.

González, K., Crozzoli, R. \& Greco, N. (2001). Utilización de enmiendas orgánicas en el control de Meloidogyne incognita. Nematología Mediterránea 29: 41-45.

Hechem, V. \& Ezcurra, C. 2006. Asclepiadaceae. En Novara, L. J. (ed.), Flora del Valle de Lerma, Serie Flora. Aportes Botánicos 7, pp. 64. Universidad Nacional de Salta.

Idárraga Piedrahita, A., Ortiz, R. D. C., Callejas Posada, R. \& Merello, M. (eds.). (2011). Flora de Antioquia 2, pp. 939. Universidad de Antioquia, Medellín.

Mahmoud, O. M., Adam, S. E. \& Tartour, G. (1979). The effect of Calotropis procera on small ruminants. II. Effects of administration of the latex to sheep and goats. Journal of Comparative Pathology 89: 251264. http://doi.org/10.1016/0021-9975(79)90064-1

Melo, M. M., Vaz, F. A., Gonçalves, L. C. \& Saturnino, H. M. (2001). Estudo fitoquímico da Calotropis procera Ait. sua utilização na alimentação de caprinos: feitos clínicos e bioquímicos séricos. Revista Brasileira de Saúde e Produção Animal 2: 15-20.

Molina Rosito, A. (1975). Enumeración de las plantas de Honduras. Ceiba 19: 1-118.

Moro, M. F., Souza, V. C., Oliveira-Filho, A. T., Queiroz, L. P., Fraga, C. N., Rodal, J. N., Araúuo, F. S. \& Martins, F. R. (2012). Alienígenas na sala: o que fazer com espécies exóticas em trabalhos de taxonomia, florística e fitossociologia? Acta Botanica Brasilica 26: 991-999.

https://doi.org/10.1590/S0102-33062012000400029

Mossa, J. S., Tariq, M., Mohsin, A., Ageel, A. M., AiYahya, M. A., Al-Said, M. S. \& Rafatullah, S. (1991). Pharmacological studies on aerial parts of
Calotropis procera. American Journal of Chinese Medicine 19: 223-231. https://doi.org/10.1142/S0192415X91000302

Nehra, O. P., Oswal, M. C. \& Faroda, A. S. (1987). Management of fodder tree in Haryana. Indian Farming 37: 31-33.

Rahman, M. A. \& Wilcock, C. C. (1991). A taxonomic revision of Calotropis (Asclepiadaceae). Nordic Journal of Botany 11: 301-308. https://doi.org/10.1111/j.1756-1051.1991.tb01408.x

Rodger, J. (1948). Indigenous ornamental plants. The Nyasaland Journal 1: 26-30.

Salako, E. A., Anjorin, T. S. \& Ulelu, A. J. (2015). Ethnobotanical survey of pesticidal plants used in Edo State, Nigeria. African Journal of Agricultural Science and Technology 3: 448-460.

Sánchez, V., Crozzoli, R. \& Greco, N. (2006). Uso de Calotropis procera para el control de Meloidogyne incognita en pepino. Fitopatología Venezolana 19: 5-9.

Sharma, G. K. (1934). Calotropis procera and Calotropis gigantea. Indian Journal Veterinary Science and Animal Husbandry 4: 63-74.

Shivkar, Y. M. \& Kumar, V. L. (2003). Anthelmintic activity of latex of Calotropis procera. Pharmaceutical Biology 41: 263-265. https://doi.org/10.1076/phbi.41.4.263.15666

Sousa Sobrinho, M., Machado Tabatinga, G., Machado, I. C. \& Lopes, A. V. (2013). Reproductive phenological pattern of Calotropis procera (Apocynaceae), an invasive species in Brazil: annual in native areas; continuous in invaded areas of caatinga. Acta Botanica Brasilica 27: 456-459. https://doi.org/10.1590/S0102-33062013000200018

XIfreda, C. C. (1984). Estudios sobre Apocynaceae argentinas III. Notas críticas sobre Forsteronia, Rauvolfia y Catharanthus. Kurtziana 17: 149-155.

Zanotti, C. A., Keller, H. A. \& Zuloaga, F. O. (2020). Biodiversidad de la flora vascular de la provincia de Misiones, Región Paranaense, Argentina. Darwiniana, nueva serie 8: 42-291. https://doi.org/10.14522/darwiniana.2020.81.878

Zuloaga, F. O., Belgrano, M. J. \& Zanotti, C. A. (2019). Actualización del Catálogo de las Plantas Vasculares del Cono Sur. Darwiniana, nueva serie 7: 208-278. http://dx.doi.org/10.14522/darwiniana.2019.72.861 